\title{
Characterization of small-scale surface topography using transmission electron microscopy
}

\author{
Authors: \\ Subarna R. Khanal, ${ }^{1}$ Abhijeet Gujrati, ${ }^{1}$ Sai Bharadwaj Vishnubhotla, ${ }^{1}$ Pawel Nowakowski, ${ }^{2}$ \\ Cecile Bonifacio, ${ }^{2}$ Lars Pastewka,${ }^{3}$ Tevis D. B. Jacobs ${ }^{1 *}$
}

\section{Affiliations:}

1. Mechanical Engineering and Materials Science, University of Pittsburgh, Pittsburgh, PA

2. E.A. Fischione Instruments, Inc., Export, PA

3. Microsystems Engineering, University of Freiburg, Freiburg, Germany

\section{Keywords:}

Surface topography; Surface roughness metrics; Transmission electron microscopy (TEM); TEM sample preparation; Self-affinity; Power spectral density (PSD)

The published version of this article can be found at: doi: 10.1088/2051-672X/aae5b3

Full citation information is as follows:

Khanal, S.R., Gujrati, A., Vishnubhotla, S.B., Nowakowski, P., Bonifacio, C.S., Pastewka, L. and Jacobs, T.D. Characterization of small-scale surface topography using transmission electron microscopy. Surface Topography: Metrology and Properties, 6, 045004 (2019)

\begin{abstract}
:
Multi-scale surface topography is critical to surface function, yet the very smallest scales are not accessible with conventional measurement techniques. Here we demonstrate two separate approaches for measuring small-scale topography in a transmission electron microscope (TEM). The first technique harnesses "conventional" methods for preparation of a TEM cross-section, and presents how these methods may be modified to ensure the preservation of the original surface. The second technique involves the deposition of the material of interest on a pre-fabricated substrate. Both techniques enable the observation and quantification of surface topography with Ångström-scale resolution. Then, using electron energy loss spectroscopy (EELS) to quantify the sample thickness, we demonstrate that there is no systematic effect of thickness on the statistical measurements of roughness. This result was verified using mathematical simulations of artificial surfaces with varying thickness. The proposed explanation is that increasing the side-view thickness of a randomly rough surface may change which specific features are sampled, but does not significantly alter the character (e.g., root-mean-square (RMS) values and power spectral density (PSD)) of the measured topography. Taken together, this work establishes a new approach to topography characterization, which fills in a critical gap in conventional approaches: i.e., the measurement of smallest-scale topography.
\end{abstract}




\section{Introduction}

It has long been known that most real-world surfaces contain surface topography across many length scales [1,2]. More recently, it has also been shown - in analytical and numerical models of single-scale $[3,4]$ and multi-scale [5,6] roughness - that a critical quantity controlling surface properties is the root-mean-square slope of the surface $h^{\prime}{ }_{r m s}$. This quantity has been directly linked to true contact area, adhesion, and friction between surfaces. Further, for a surface with multi-scale roughness, the smallest-scale features dominate $h^{\prime}{ }_{r m s}$. This is shown schematically in Fig. 1a, and is shown mathematically in the equations for computing $h^{\prime}{ }_{r m s}$ from a real-space or frequency-space description of a surface [6,7]. However, conventional surface measurement techniques (such as stylus or optical profilometry, and atomic force microscopy) are incapable of reliably measuring topography at the smallest lateral length scales, primarily due to tip-size artifacts and noise in the measurements [8]. Therefore, it is impossible to accurately measure this critical quantity $h^{\prime}{ }_{r m s}$ with conventional techniques. Instead, new approaches are required for measuring surface topography at the smallest scales.
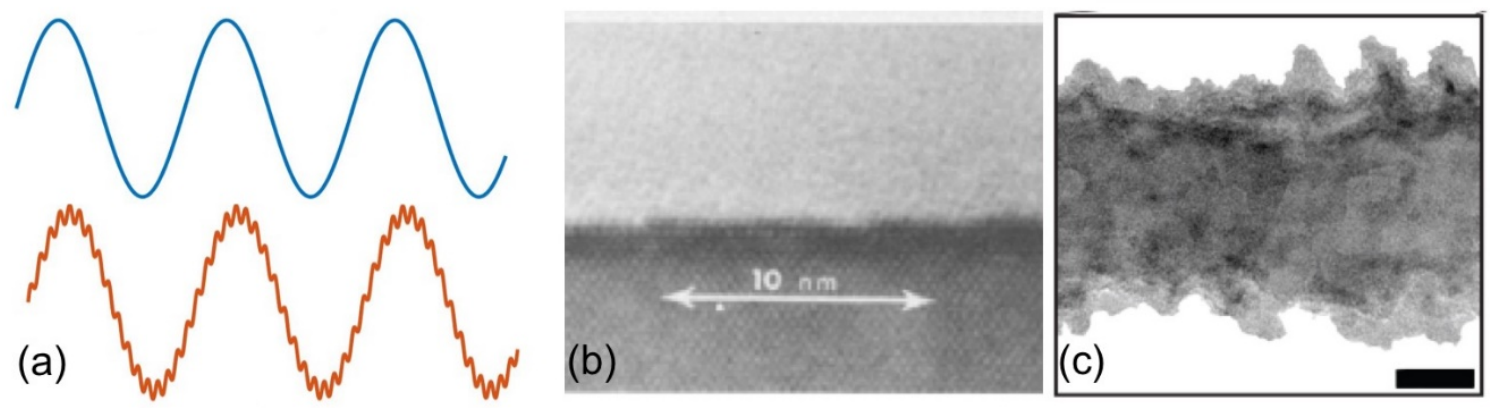

Figure 1: The smallest-scale roughness has the biggest impact on local surface slope; yet it is hardest to measure. A simple schematic (a) shows a single sinusoid (top) with a root-mean-square slope of 0.5 , and the superposition of a second sinusoid with a smaller amplitude $\left(1 / 10^{\text {th }}\right.$ of the original $)$ and a smaller wavelength $\left(1 / 15^{\text {th }}\right.$ of the original), which raises the root-mean-square slope to 1.0. Early TEM measurements (b) of the boundary between single-crystal silicon and its oxide were used to measure the topography of interfaces. More recent TEM measurements (c) enable the characterization of free-surface topography, but only on nanoparticles or nanowires that are electron transparent by nature. The scale bar in (c) is $20 \mathrm{~nm}$. Image (b) reproduced from Ref. [9], copyright The Electrochemical Society, 1987. Image (c) reproduced from Ref. [10], copyright American Chemical Society, 2012.

The present work builds on prior TEM investigations into the roughness of interfaces (Fig. 1 b,c). For example, the roughness of a native oxide grown on silicon has relevance for its electronic properties, and was first studied using cross-section TEM forty years ago [11,12]. Many early investigations reported only the maximum height variation observed along the interface, and correlated this with growth conditions [9]. However, Goodnick and coauthors [13] went further, digitizing the interface contour, and thus enabling its statistical characterization. The authors computed scalar roughness parameters, such as the root-mean-square height fluctuation and the 
correlation length. This pioneering work on the characterization of native silica roughness has been widely adopted in the study of interfaces and understanding its effect on properties (for a more recent example, see Ref. [14]). It has also been applied to the TEM characterization of surface roughness in nanowires [10], which are electron transparent in their native state. However, this same framework has not been widely applied to the general study of surface topography of engineered components. This lack of use likely stems primarily from the difficulty of preparing a free surface for TEM examination. There are two typical approaches for preparing a thin section for TEM examination, as discussed in the next paragraphs; both can significantly alter or destroy the topography of the free surface.

The first common technique, the focused ion beam (FIB) lift-out, uses a FIB to mill two adjacent trenches with a free-standing thin lamella in between. Then, a nanomanipulator is used to transfer this lamella to a TEM grid, where it is further thinned to electron transparency. While this technique is efficient and extremely widely used for materials analysis, it cannot be done without altering the original surface. The ion beam hits the surface at a nearly perpendicular orientation, and is known to cause a cascade of collisions that removes and reorganizes the near-surface material [15]. For typical cross-section preparation, this ion-beam damage is avoided by depositing a protective layer (typically platinum) over the region of interest. However, the process of depositing platinum still exposes the surface to direct impingement of the ion beam (albeit briefly) which can cause damage. Further, the presence of the high-atomic-number protective metal layer strongly scatters electrons, thus reducing the maximum thickness for TEM transparency and also causing shadowing of the free surface in the final TEM examination. For these reasons, typical FIB lift-out cross-sections are not well-suited for the TEM examination of surface topography.

Second, the so-called "conventional" cross-section preparation involves grinding and glancingangle ion milling. The conventional technique is well documented elsewhere (for example, Refs. $[16,17])$. Briefly, it includes sandwiching bulk pieces of the material together, then cutting and grinding a thin circular disc which contains the region of interest. Then, using dimple-grinding and low-angle ion milling, the region of interest is thinned down until perforation, rendering the nearby material electron transparent. (A modified version of this process is discussed in detail in the Experimental Methods section of the present article.) When performed in its normal fashion, the outermost surface can be removed or damaged during the grinding and low-angle ion milling.

In addition to the damage of the free surface from sample creation, an additional challenge of TEM topography measurement arises because the TEM produces a 2D projection of a 3D structure. Goodnick et al. [13] discuss the effect of projection for a $\mathrm{Si} / \mathrm{SiO}_{2}$ interface and delineate two considerations. First, a 2D surface is reduced to a 1D line contour, and thus a mathematical model is required to convert from 1D statistics to 2D statistics. This can be readily accomplished by assuming self-affinity and isotropicity of the surface (as done by Goodnick et al.) and as described 
explicitly using a power spectral density representation of the surface in Ref. [8]. The second consideration of measuring topography from the 2D TEM projection is that the line contour measured on a sample of non-zero thickness may differ from a "true" line contour that would be measured from an infinitely thin slice through the surface. Goodnick et al. estimated the effect of thickness on two scalar parameters: correlation length and RMS roughness. They used computergenerated random surfaces with Gaussian or exponential spatial correlation and cross-section thicknesses that were large compared to the chosen correlation lengths. Their mathematical results showed a monotonic reduction in measured correlation length and RMS roughness with crosssection thickness. However, the authors did not directly measure the thickness of their experimental cross-sections and so these mathematical results have not been experimentally verified. Further, many real-world surfaces have multi-scale roughness with self-affine character and thus do not fall within the computed regime.

Overall, to achieve the complete characterization of surface topography in ways that can be quantitatively linked with surface properties, new approaches are required for characterizing free surfaces at the smallest scales. While the TEM has been used previously to measure interface roughness, the only TEM analysis of free-surface topography has been on nanoparticles and nanowires, which are electron transparent by nature. Using the TEM to characterize surface topography of general surfaces presents two difficulties: the preservation of the free surface during TEM sample preparation; and the analysis of the surface contour once it is created, especially with the question of how the TEM's 2D projection affects the measurement of topography. The present article introduces two surface-preserving techniques for preparation of TEM samples for topography characterization (Sect. 2), and directly investigates the effect of thickness on measured results (Sect. 3).

\section{Sample preparation for topography measurement in the TEM}

The preparation of samples is important for all TEM measurement, but it is particularly critical for topography characterization. Two different approaches are presented in this manuscript: (1) a modified version of the "conventional" cross-section preparation technique, which we call the surface-preserving cross-section technique; and (2) a simple deposition of the surface of interest onto a thin-wedge substrate that is already electron transparent, which we call the wedge deposition technique. The first technique is generally applicable to all materials; the second technique can only be used for materials that can be deposited or grown in a thin layer. In the present investigation, both techniques were performed on the same material to facilitate comparison.

\section{Material for investigation}

The surface topography characterization was performed on an ultrananocrystalline diamond (UNCD) film. Conductive (boron-doped) UNCD was deposited (by Advanced Diamond Technologies, Romeoville, IL) with hot-filament chemical vapor deposition (HFCVD), as 
described in Ref. [18]. The gas mixture was H-rich, with a chamber pressure of 5 Torr and a substrate temperature of $750{ }^{\circ} \mathrm{C}$. The ratio of boron to carbon was maintained at $0.3 \mathrm{at} \%$, to achieve high conductivity in the material. In the same batch, films were deposited both on polished silicon wafers as well as on microfabricated wedge samples (Silicon wedges, Bruker, Billerica, MA).

\section{Sample creation for bulk materials: The surface-preserving cross-section technique}

The "conventional" cross-section preparation process is well described elsewhere [17], and so is only briefly reviewed here. The process is shown schematically in Fig. 2. For bulk materials, two surface-containing pieces of material are removed from the bulk, typically using a low-speed saw or through a controlled fracture process. For the present samples, which were supported by a silicon wafer substrate, two $4 \times 5$-mm pieces were cut out using an ultrasonic disk cutter (Model 170, Fischione Instruments, Export, PA). These two small pieces were sandwiched, with the surfaces of interest facing each other, with additional "dummy" silicon wafers added to increase the top-to-bottom thickness. A carbon-based adhesive secures the pieces together, and also prevents the surfaces of interest from making direct mechanical contact and damaging one another. Once the structure is bonded, a cylindrical core ( $3 \mathrm{~mm}$ in diameter) is extracted using the same ultrasonic disk cutter with a circular cutting bit. This cylindrical core is inserted into a brass tube and is then sliced into thin $(\sim 0.5 \mathrm{~mm})$ discs using a wafer saw. These discs are ground (using grits of 600 to 1200) and polished (using 6- $\mu \mathrm{m}$ to $1-\mu \mathrm{m}$ diamond lapping) to a thickness of $0.1 \mathrm{~mm}$ using a specimen grinder tool (Model 160, Fischione Instruments). These thin disks were then dimpled using a dimpling grinder (Model 200, Fischione Instruments) to a minimum thickness of approximately $10 \mu \mathrm{m}$. Finally, glancing angle ion milling (Model 1050, Fischione Instruments) is used to only just achieve perforation of the sample in region of interest.

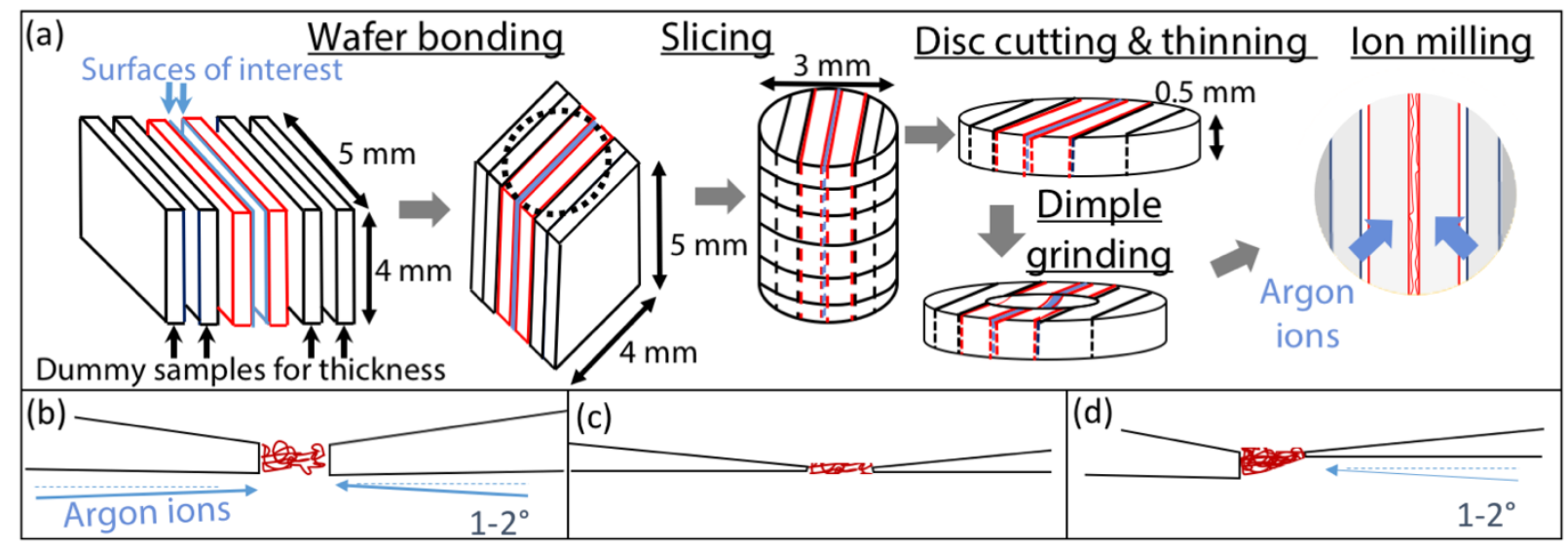

Figure 2: For bulk samples, a cross-section must be prepared for TEM imaging, but with extra care to preserve the original surface. The so-called "conventional" cross-section preparation is shown schematically in panel (a). The principal modifications for surface-preserving cross-sections include: the use of low-energy, ultra-low-angle ion milling (b); the characterization of a region which never fully perforates, and still has some adhesive present (c); and optionally, the use of single-side milling to ensure that one free surface is totally free from direct impingement of ions (d). 
There are two sets of slight modifications required for the creation of surface-preserving crosssections. First, extra care is required during the ion milling process to avoid damaging the free surface. This was achieved by applying a low incidence milling angle of $2^{\circ}$ during thinning (Fig. 2b), in contrast to the typically used angle of $5^{\circ}$ or higher. Because of this small angle, the thinning can only proceed from the non-dimpled side because the dimpled side has a steeper angle and the region of interest will be shadowed. Further, a lower energy of ions was used for the final thinning steps ( $2 \mathrm{keV}$ instead of typically used $4 \mathrm{keV}$ ) and the sample condition is checked more frequently. These modifications add time but ensure that thinning does not proceed too rapidly and damage or destroy the free surface. Second, while the sample is normally milled all the way until perforation in the region of interest, here the region of interest was thinned down to near perforation, to ensure minimal exposure of the free surface to the ions. This was achieved by attaining perforation in a location adjacent to the region of interest, and then carefully increasing the size of the perforated hole until it approached (but did not reach) the region of interest. The characterization can then be performed only in regions where the adhesive remains intact (Fig. 2c), or where the adhesive has only just been removed. In the former region, the original surface has never been directly exposed to the ion beam; in the latter region, the ion exposure of the surface is minimal. For especially beam-sensitive materials, single-side ion milling can be performed (Fig. $2 \mathrm{~d}$ ); which ensures that the ions can never impinge directly on the region of the free surface that is being examined.

\section{Sample creation for thin-film materials: The wedge deposition technique}

For thin-film materials that can be grown or deposited, the preparation for TEM is simpler because they can be applied to a substrate that is already electron transparent. In general, such a substrate can be microfabricated, e.g., by anisotropic etching of silicon, or can be made by purchasing and/or modifying a commercial TEM specimen grid. In the present investigation, the $2-\mu \mathrm{m}$-thick UNCD film was applied to a commercial substrate (Hysitron Picoindenter wedge substrates, Bruker, Billerica, MA). These substrates have a microfabricated silicon wedge that is several millimeters in length, with a thickness that tapers from several microns at the base to either 1 micron or 100 $\mathrm{nm}$ at the apex (Fig. 3). This geometry provides a long region that is electron transparent, while the flat plateau enables the approximation of deposition on a flat substrate. For films with large residual stresses or other geometry dependence, the thicker 1- $\mu \mathrm{m}$ plateau is recommended, as the larger lateral area provides more mechanical constraint. However, for the present work, the UNCD films were deposited using a process specifically designed to reduce residual stress (as discussed in Ref. [18]); therefore, the narrow 100-nm plateau was used to minimize the through-thickness for optimal imaging. 


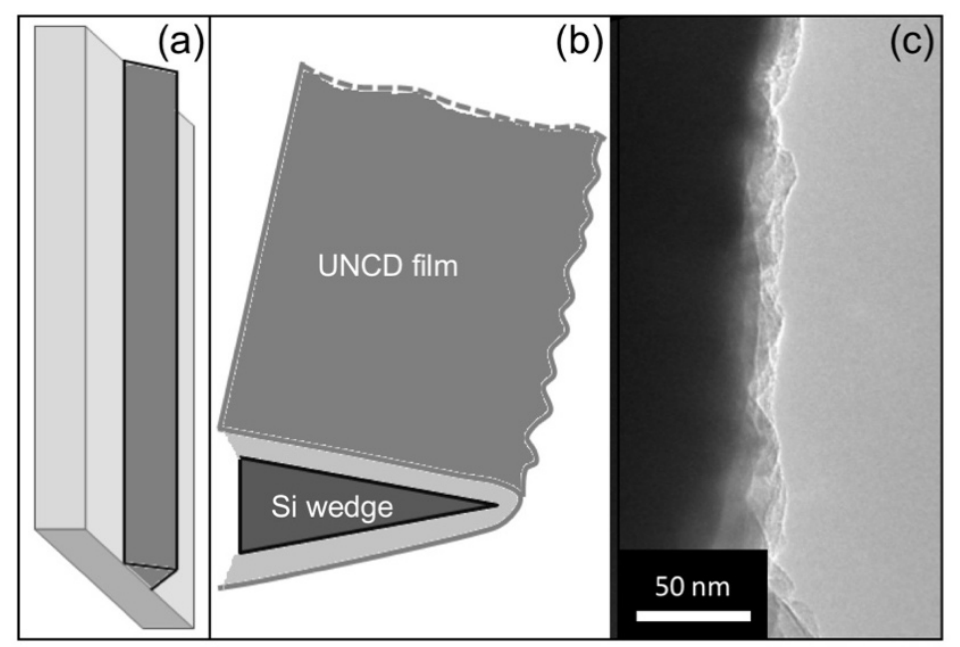

Figure 3: For deposited materials, a pre-fabricated thin-wedge substrate provides a simple route to TEM sample preparation. A commercial substrate was used (a) which contains a large (mm-scale) flat carrier chip with a long, narrow microfabricated wedge. The tapered wedge was coated with the UNCD material of interest (b) and then imaged in profile in the TEM (c).

\section{TEM imaging and extraction of quantitative surface contours}

Both the cross-section and wedge samples of UNCD were imaged in a TEM (2100F, JEOL, Tokyo, Japan) at an accelerating voltage of $200 \mathrm{keV}$. Before collecting images, a brief damage study was performed to determine whether there is a threshold of exposure dose at which the surface topography will be modified by the electron beam. In general, the beam sensitivity of the material will vary with chemistry and structure as well as accelerating voltage and should be checked in all investigations. In the present results, the UNCD surface showed no variation over time for the exposure dose rates used (up to a maximum of $1900 \mathrm{e}^{-/} \AA^{2} / \mathrm{s}$ ). Images were collected at various magnifications from $100 \mathrm{kx}$ to $500 \mathrm{kx}$, in a variety of locations on all samples. Surface profiles were extracted from the TEM imaging by tracing the contrast change at the boundary of the material. The surface of interest is often the outer boundary of the sample (as it is in Fig. 3b) and is therefore easily distinguished from the background. As discussed in the next section, there is sometimes a layer of amorphous carbon-based adhesive over the surface of interest; in these cases, the boundary can be distinguished either by crystallinity (using direct observation of the lattice, or by Fourier filtering of the image, or using dark-field imaging) or by chemistry (using annual darkfield STEM, energy dispersive x-ray spectroscopy, electron energy loss spectroscopy, energyfiltered TEM, etc.). In some cases, including those with large contrast variation, the boundary can be determined using standard edge-detection algorithms such as the built-in algorithms in MATLAB or ImageJ. In other cases, these algorithms were found to be inaccurate at the highest magnifications due to smaller contrast variations between the material and the background, and also due to contrast variation inside the material (from the atomic lattice). In these latter cases, and 
in the present analysis, the profiles were extracted using custom MATLAB scripts that enable manual point-picking and conversion to a set of $\mathrm{x}-\mathrm{y}$ positions.

Quantitative measurement of thickness for cross-section and wedge samples using electron energy loss spectroscopy

In addition to imaging the sample and extracting its topography, as described above, it is often useful to understand the thickness of the measured sample. This is readily done in the TEM using EELS, which was performed in this investigation using STEM mode operating at $200 \mathrm{kV}$. The thickness of a sample can be determined by measuring the fraction of electrons that are inelastically scattered by the sample [16]. For a thicker sample, the electrons pass through more atoms and more electron clouds and have a higher likelihood of knocking out a valance or core shell electron from the sample. The thickness $t$ can be determined from the EELS spectrum as follows [19,20]:

$$
t / L_{M F P}=\frac{\ln \left(I_{\text {tot }}\right)}{\ln \left(I_{0}\right)}
$$

where $I_{0}$ is the intensity (integrated number of detector counts) associated with the zero-loss peak, $I_{\text {tot }}$ is the intensity of all collected electrons, and $L_{M F P}$ is the total inelastic mean free path for all types of inelastic scattering. Because the UNCD is primarily composed of $\mathrm{sp}^{3}$ carbon [18], the $L_{M F P}$ for carbon in the diamond cubic structure is used, which is $112 \mathrm{~nm}$ [20].

\section{Results and Discussion}

Surface profiles and roughness parameters measured using TEM

Surface profiles were measured and extracted using 168 different TEM measurements of the UNCD film at various locations and magnifications. The characterized UNCD samples fell into three types: (1) samples created using the surface-preserving cross-section technique, where the adhesive had only just been removed (Fig. 4a); (2) samples created using the surface-preserving cross-section technique, but where the adhesive that glued the samples together was extremely thin but still covered the surface (Fig. 4b); and (3) samples created using the wedge deposition technique (Fig. 4c). 


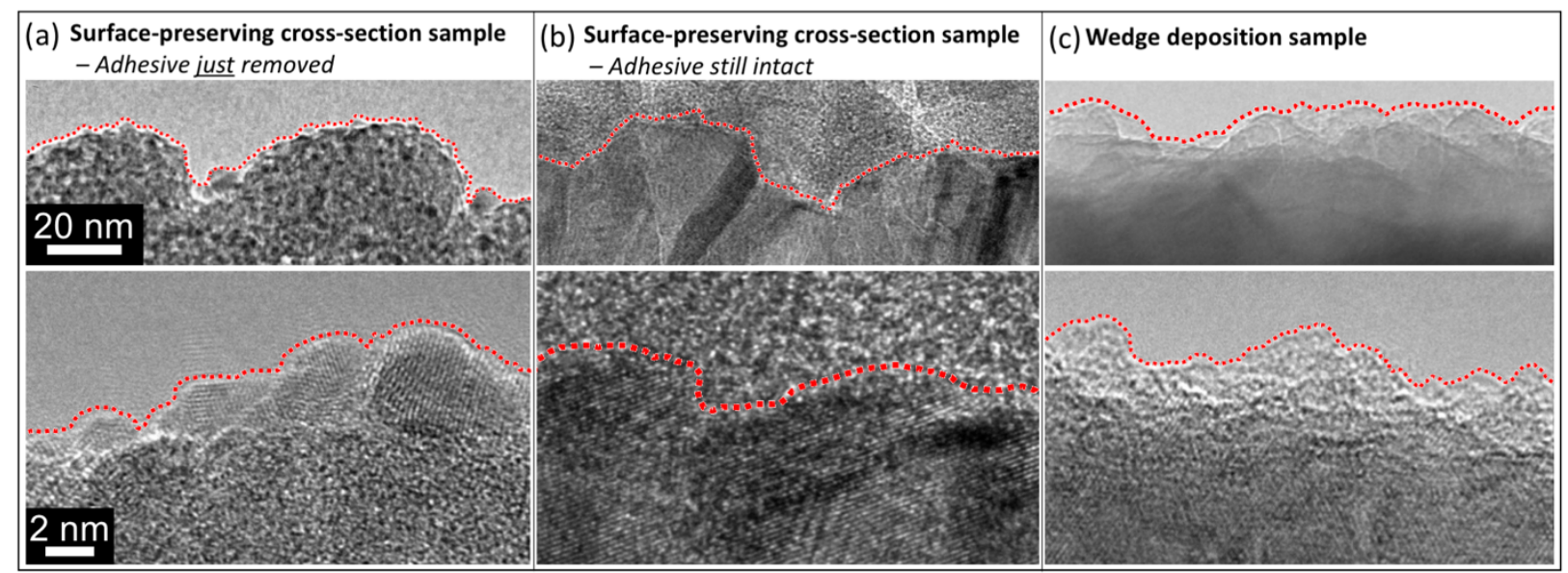

Figure 4: Using both sample preparation approaches, the topography can be directly extracted using the contrast in the TEM images. TEM images were captured at low magnification (top row, all with a common scale bar) and at high resolution (bottom row, common scale bar). Line contours of the topography of the free surface were extracted using algorithms to trace the boundary of the material when viewed in profile. As discussed in the main text, samples were prepared using surface-preserving cross-section sample preparation (ab), and by depositing material on a premade electron-transparent substrate (c). For the surface-preserving crosssection samples, the topography was imaged both in regions where the adhesive was only just removed (a) and also in regions where the adhesive was still intact and covering the sample (b). Both conditions ensure minimal exposure of the free surface to the ion beam and therefore minimal modification from its native state. For the regions where the adhesive was still intact (b), the boundary was readily determined as the UNCD has visible atomic lattice and a darker contrast than the amorphous adhesive.

In order to quantitatively assess and compare results across sample preparation techniques, two types of roughness descriptors were computed: a statistical descriptor in the form of the power spectral density $C$; and root-mean-square (RMS) parameters, including the RMS height $h_{r m s}$, RMS slope $h^{\prime}{ }_{r m s}$, and RMS curvature $h^{\prime}{ }^{\prime}{ }_{r m s}$. The power spectral density is the Fourier transform of the height autocorrelation function, and distinguishes the contribution to topography from different length scales or wavelengths $\lambda$. The PSD was computed according to the best practices described in Ref. [8], and is presented as a function of wavevector $q=2 \pi / \lambda$. Figure 5 a shows the averaged PSD for all samples of each preparation. When the small-scale portion of the curve $(\lambda<20 \mathrm{~nm})$ is fit using a power-law function, the scaling exponents are $-2.87 \pm 0.08$ for the wedge deposition sample, and $-2.85 \pm 0.12$ and $-2.97 \pm 0.16$ for the cross-section samples (adhesive intact, and adhesive removed, respectively). From this the Hurst exponent of the surface can be calculated (as described in Ref. [21]) as $H=0.93 \pm 0.04,0.92 \pm 0.06$, and $0.98 \pm 0.08$, respectively. (Note that the wedge samples are the same ones that were characterized in Ref. [21], which used the wedgedeposition method, exactly as it is described in the present article. As noted in that prior article [21], the precise value of Hurst exponent that is measured depends on the range of wavevectors over which the fitting is done.) The measured Hurst exponents between the different sample preparations are identical within experimental uncertainty. This finding demonstrates that 
the statistics of the measured surface do not depend on preparation, whether wedge deposition technique or surface-preserving cross-section technique - and in the latter case, whether the adhesive is left intact or just barely removed.

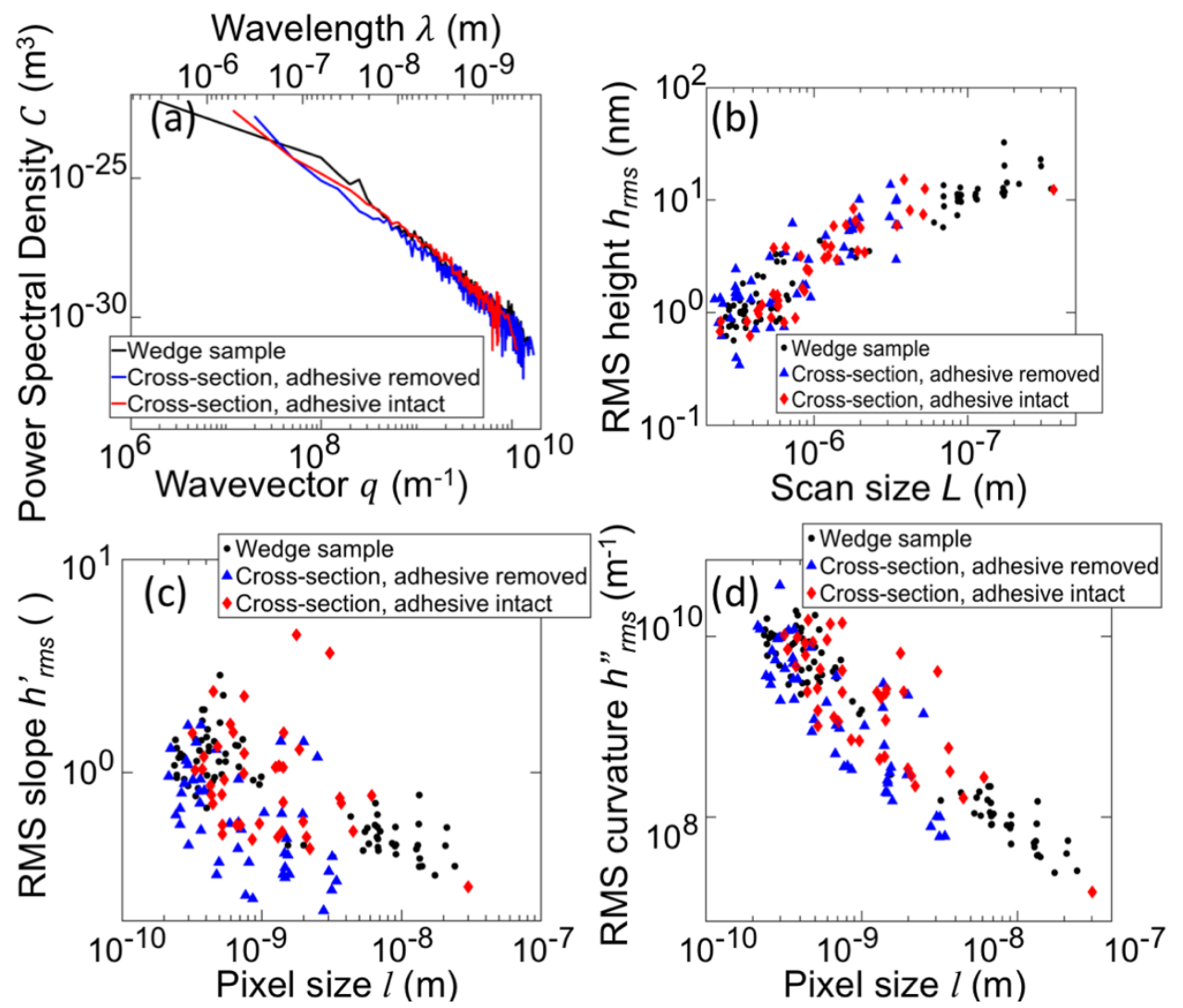

Figure 5: The statistics of roughness are measured at the smallest length scales, and are not affected by the method of sample preparation. Roughness statistics were measured from 168 different TEM images (similar to those shown in Fig. 4 and Supp. Fig. S1), including various locations and various magnifications for each type of sample. The PSD of topography (a) contains a statistical picture of the contributions to roughness from different lateral size scales. Here, an averaged PSD has been stitched together from the individually measured PSDs computed from each image (using the techniques described in Ref. [21]). The PSDs demonstrate that the material can be characterized at size scales below $10 \mathrm{~nm}$ (above $q=6 \times 10^{8} \mathrm{~m}^{-1}$ ); these scales are inaccessible using conventional techniques. The root-mean-square height (b), slope (c), and curvature (d) of topography are important scalar descriptors of the surface, which are used in many mechanics models of roughsurface properties. Their values are known to depend on measurement size, and here they are measured at various magnifications, including down to the Ångström scale. For all four roughness descriptors, the measurements are indistinguishable across sample preparation techniques.

Scalar descriptors of roughness were computed from the real-space topography measurements using the following equations:

$$
h_{\mathrm{rms}}^{2}=\frac{1}{L} \int_{0}^{L} h^{2}(x) d x, \quad h_{\mathrm{rms}}^{\prime 2}=\frac{1}{L} \int_{0}^{L}\left(\frac{d h}{d x}\right)^{2} d x, \quad h_{\mathrm{rms}}^{\prime \prime 2}=\frac{1}{L} \int_{0}^{L}\left(\frac{d^{2} h}{d x^{2}}\right)^{2} d x
$$


The computed values vary significantly depending on scan size and pixel size, as shown in Ref. [21], and therefore must be presented in this context. The RMS height is shown as a function of scan size $L$ in Fig. 5b, because the largest-scale features have the most significant effect. The RMS slope (Fig. 5c) and RMS curvature (5d) are presented as a function of pixel size, because these parameters depend most strongly on the small-size features. (Note that "pixel size" is determined by the resolution of the image and may not necessarily correspond to the total image size divided by the number of pixels in the camera. Therefore, in the present results, the pixel size corresponds to the average spacing between measured points in the profile.) In all cases, there is scatter in the data from sample to sample, but at a given scan size there is no consistent trend with the type of sample preparation. The purpose of the present report is to compare and assess the measured results between different techniques of TEM preparation; for comparison of results against other methods of topography measurement (AFM, stylus profilometry), see Ref. [21]. In short, the TEM-measured data is commensurate with AFM measurements of the same material, but provides small-scale roughness information that is not accessible using AFM.

These results imply that both of the sample preparation approaches described in Sect. 2 are equally valid for topography examination. For bulk materials, there is no choice except to use the more time-consuming cross-section preparation. However, for deposited materials (including many technologically relevant materials, such as films and coatings) the wedge deposition technique represents a facile method for topography evaluation. This technique can be used quite broadly in materials deposition, simply by including an electron transparent wedge as a witness chip that is co-deposited with any other material of interest. After deposition, the topography of the witness chip can be straightforwardly evaluated in the TEM to represent the topography of the flat sample.

\section{Investigation into the effect of thickness on experimental results}

It seems possible that the through-thickness of a TEM sample will have a strong effect on the measured topography, because samples with different thicknesses may cause different features to contribute to the observed surface profile. Therefore, in the present study, the effect of thickness was directly investigated using the analytical capabilities of the TEM. For each location where topography was measured (Fig. 6a), EELS data was collected (Fig. 6b). The thickness at each of these locations is determined using Eq. 1. The thicknesses varied between samples from $28 \mathrm{~nm}$ to $150 \mathrm{~nm}$ for the cross-section samples. For the wedge samples, the thickness at the surface varied from $14 \mathrm{~nm}$ to $59 \mathrm{~nm}$, and because of the rounded apex of the wedge, the thickness increased rapidly with depth into the material. By $10 \mathrm{~nm}$ below the surface, the throughthickness was between 80 and $120 \mathrm{~nm}$. Figure $6 \mathrm{c}$ and $6 \mathrm{~d}$ directly present the PSD and RMS height as a function of sample thickness. The PSD is indistinguishable between all samples, regardless of thickness. The computed RMS height does show variation between samples, but exhibits no systematic effect of thickness. Likewise, the RMS slope and curvature show no trend with thickness (Supplementary Figure S2). Because it is only the outermost contour of the UNCD that contributes to the measured profile (red line in Fig. 4), and because the method of 
preparation of the sample does not affect the measured statistics, then it seems unlikely that other parameters of the sample preparation will have a strong influence on the final result. For instance, factors such as the precise taper angle of the cross-section sample, or the thickness of the thin film in the wedge deposition sample (which determines the radius of curvature of the outermost edge), seem unlikely to affect the measurements. This assumption is specifically tested in the following sub-section by systematically varying the radius of curvature of a simulated wedge sample. Overall, the present TEM-based surface topography measurements have no dependence on the type of preparation used, nor the final thickness of the sample; therefore, they likely represent the true topography of the original surface at these smallest scales.
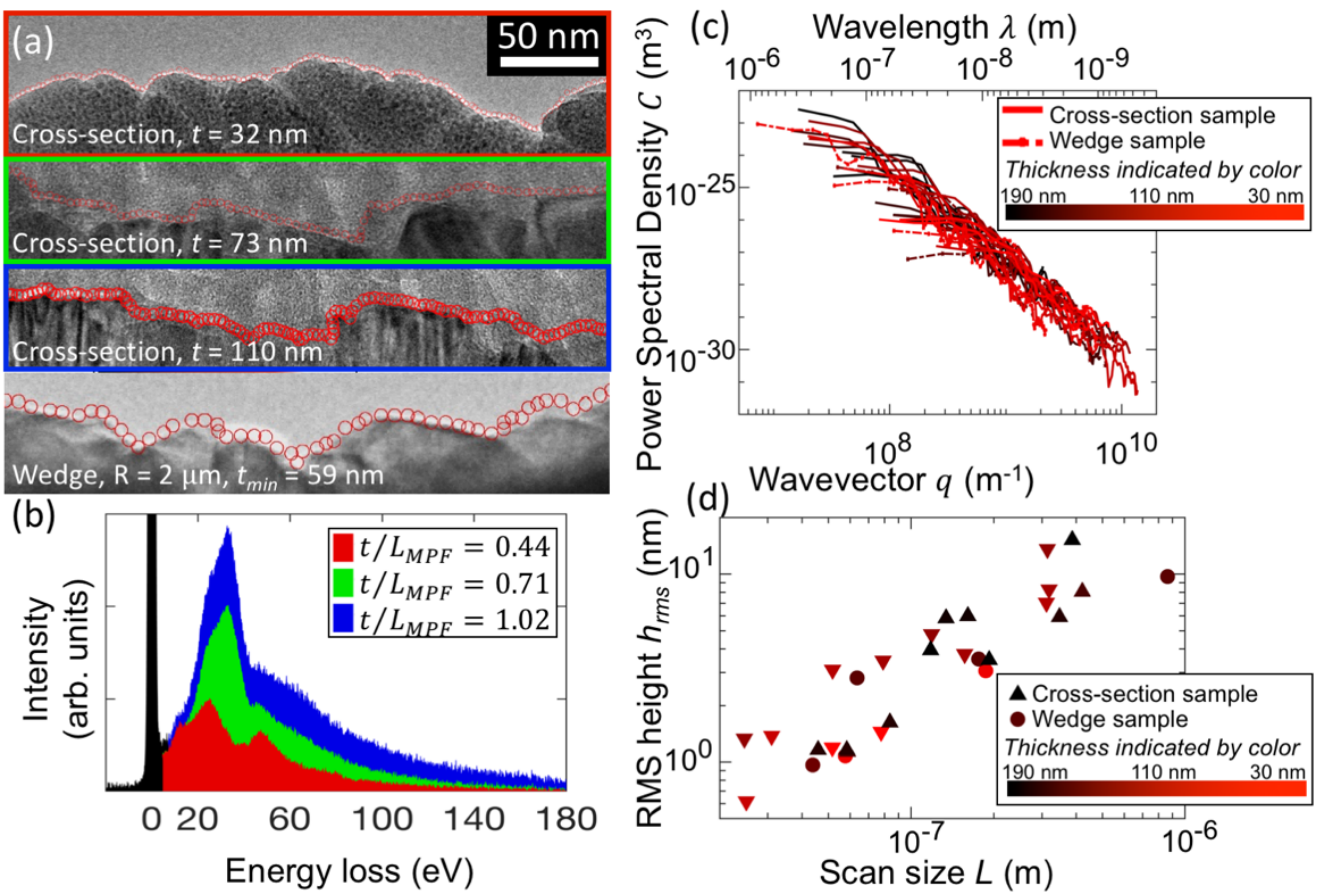

Figure 6: Direct measurements of thickness using analytical TEM demonstrate no systematic effect of thickness on results. Using electron energy loss spectroscopy, the thickness was measured (see main text) for a subset of the profiles from Fig. 5. Four representative profiles are shown (a), from cross-sections (with and without adhesive removal) and from a wedge sample. The corresponding EELS spectra for the cross-section samples (b) show the intensity as a function of energy loss. The thickness is determined from the ratio of zeroloss intensity (black region) to total intensity (sum of black and colored areas). The images in (a) share a common scale bar, and the outline color corresponds to the colored plots in (b). Plots of PSD (c) and RMS height (d) show random variation within the measurements but show no systematic trend with increasing sample thickness. RMS slope and curvature (Supplementary Figure S2) also show no trends with thickness.

\section{Measuring the effect of thickness using artificial surfaces}

While the experimental trends are robust, the sample size is not as large as it would ideally be to draw firm conclusions. To more systematically evaluate the effect of thickness on measured 
results, artificial (computer-generated) self-affine surfaces were created and evaluated. First, we created an artificial random, self-affine surface using a Fourier filtering algorithm, as described in Refs. [8,22]. The surface has a resolution of $4000 \times 4000$ pixels spanning a size of $400 \mathrm{~nm} \times 400$ $\mathrm{nm}$. It is self-affine across all scales with a Hurst exponent of $\mathrm{H}=0.8$. The surface was created such that its RMS slope is unity, $h_{\mathrm{rms}}{ }^{\prime}=1$. The corresponding surface had roughness in the range of single-digit nanometers; however, the following analysis is scale-independent and is expected to work equally well for self-affine surfaces that are significantly rougher or smoother (i.e., those that have larger or smaller values of Hurst exponent and RMS slope). We also note that this analysis is intended for isotropic, randomly rough surfaces; a separate study would be required to understand the behavior of anisotropic surfaces with periodic or regular structures that depend on orientation.

Two types of TEM-analogous profiles were created and analyzed. First, to approximate the crosssection samples, long strips of varying thickness were extracted from the overall surface. More specifically, we chose a random position on the surface and extracted strips of the surface, centered on this position, with various thickness $t$ along the y-axis, as shown schematically in Fig. 7a. The sampled profile was generated for each strip by finding the maximum vertical height $h$ at each $x$ position along the strip. In this way, the lateral projection of the strip was determined and used as a TEM-analogous profile (Fig. 7b) for various values of $t$. Second, to approximate the wedge deposition samples, a similar approach was used but with the superposition of a radius $R$ of curvature along the through-thickness direction, as shown in Fig. 7e. Representative sampled profiles for different strip thicknesses and different wedge radii are shown in Fig. $7 \mathrm{~b}$ and $7 \mathrm{f}$, respectively. Finally, the power spectral densities of these sampled profiles were computed. To remove bias from the calculations, profiles of the random surfaces were sampled at 10 different and non-overlapping locations and the resulting PSDs were averaged (Fig. 7c and 7g). These results demonstrate no variation in the measured power spectral density with variations in sample thickness. 

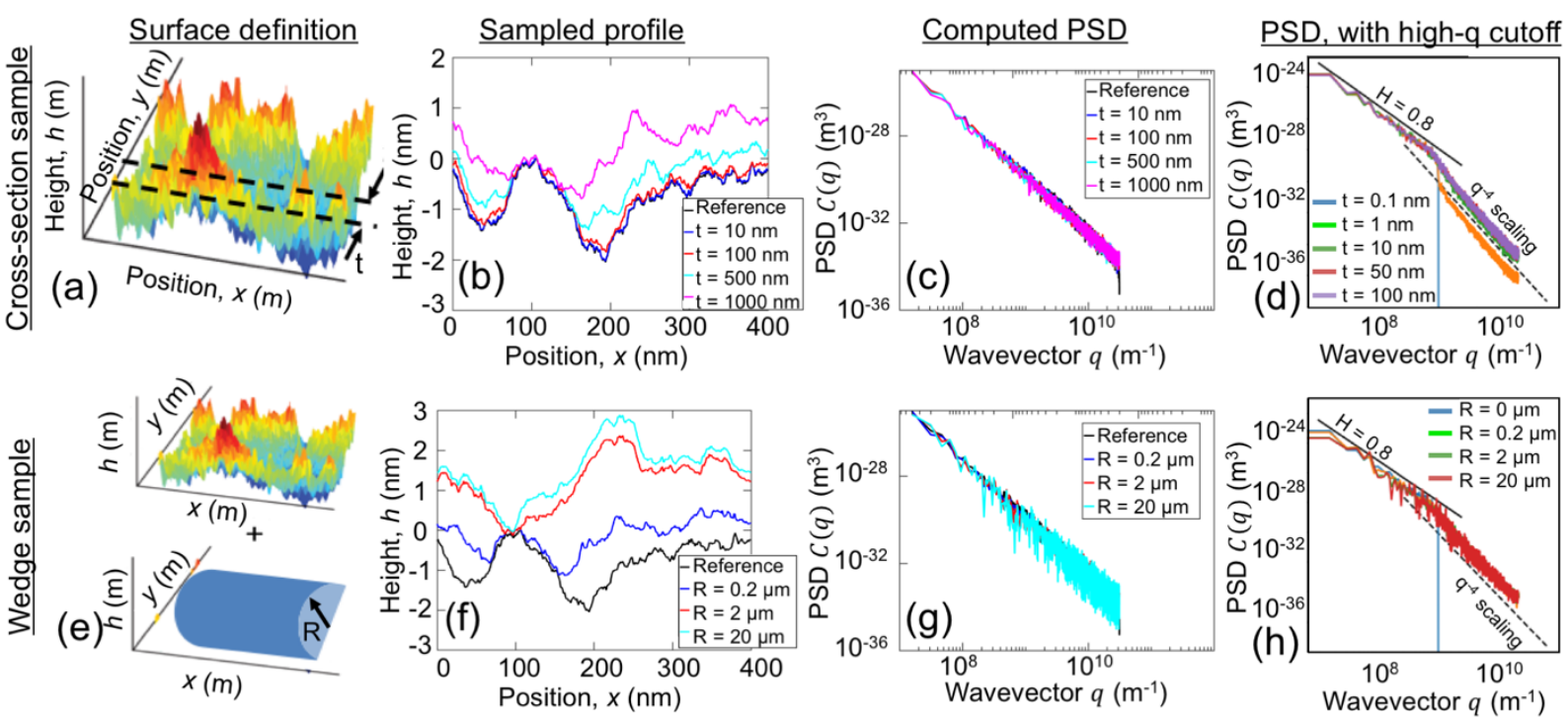

Figure 7: Measuring the effect of thickness on the statistics of roughness for artificially-created surfaces. A random self-affine surface was computer-generated and sectioned to a desired thickness (a). This process was repeated to create line contours (b) with variable thickness $t$. For self-affine surfaces, the computed power spectral densities (c) were shown to be completely independent of thickness of the profile. When a minimum wavelength of roughness was introduced (see main text) at $5 \mathrm{~nm}\left(\mathrm{q}=1.26 \mathrm{~nm}^{-1}\right)$, then the thicker samples do show an effect, manifesting as $\mathrm{q}^{-4}$ scaling behavior, which was never observed experimentally. The process was repeated (e-h) to simulate the wedge deposition surfaces. For various values of cylinder radius $R$, the results were the same as the cross-section simulations.

As demonstrated in the profile images of Fig. 7(b,f), there are certainly changes in the particular surface features that contribute to the sampled profile, but because the newly-appearing surface features have the same statistical character as the disappearing surface features, there is no net change in the statistical character of the measured surface. We note that these findings are in contrast to the results obtained for surfaces with Gaussian or exponential height-height correlations investigated in Ref. [13].

To further investigate the difference from earlier models, we introduced a characteristic lengthscale to the simulated profiles in the form of either a short-wavelength cut-off or a long-wavelength roll-off. The short-wavelength cut-off means that there is no contribution to overall roughness from wavelengths smaller than a certain size, and the profile will be smoothly varying if examined at smaller size-scales. As shown in Fig. 7d,h, this introduced a spurious contribution to the PSD that scaled as $q^{-4}$ (corresponding to an apparent Hurst exponent of 1.5), similar to the effect of tip radius in atomic force microscopy images [8,23]. However, such a transition was not observed in any of the experimental data. Instead, the PSD is observed to be approximately self-affine all the way to the highest measurable $q$. Additionally, the real-space images clearly show Ångstrom-scale variations in topography that contradict the idea of a length scale at which the profile becomes smooth. The effect of a low- $q$ roll-off was also tested (Supplementary Figure S3), and manifests 
as a reduction in magnitude of the PSD at wavenumbers below the cutoff (i.e., large wavelengths). This roll-off effect is deemed less significant because large-wavelength differences are easily detectible using AFM and do not require the high resolution of a TEM-based technique.

\section{Combining insights from experimental and simulation results}

The experimental and simulation measurements taken together demonstrate that the thickness and preparation of the sample does not affect the measured statistics of surface topography. From a practical perspective, there will be two maximum thresholds of thickness that should be considered for TEM-based topography measurements. First, the maximum thickness of the sample is bound by the need for electron transparency to enable TEM imaging. The mean free path of an electron depends on the material it is passing through and, for example, is several times larger for carbon than it is for heavy elements like gold [16]. Therefore, the thickness of the measured portion of the sample (red lines in Fig. 4) should be either comparable or less than the length of the mean free path in that material. Fortunately, a violation of this condition is easily observed in the TEM as a significant reduction in resolution in the region of interest. This can be remedied by further thinning in the ion mill (for the cross-section method) or by deposition of a thinner film of material (for the wedge deposition technique). Second, if the material itself shows "roll-off" behavior (where the PSD is flat below some critical value of $q$ (i.e., above some critical length scale $\lambda_{\text {roll- }}$

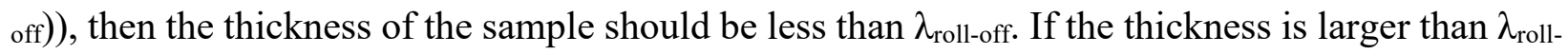
off, there will be a reduction in the magnitude of the measured PSD. However, in our experience and that of several other authors (e.g., Ref. [24]), the roll-off region of most real surfaces begins in the range of $\lambda>1-100 \mu \mathrm{m}-$ a larger thickness than is practical for use in the TEM. Furthermore, it should be noted that the roll-off behavior was still adequately detected at larger thicknesses, just with a reduced magnitude of the PSD at smaller $q$. Therefore, the TEM data can be paired with atomic force microscopy data (e.g., Ref. [21]), which will more accurately characterize the largerscale topography. Overall, for the vast majority of real-world materials, the described methods will enable the accurate measurement of roughness statistics with no significant effect of sample thickness.

\section{Conclusions}

In conclusion, the present work demonstrates the use of transmission electron microscopy to obtain the critical small-scale topography characterization that is inaccessible using conventional techniques. Two separate sample preparation techniques are presented including the preparation of a surface-preserving cross-section and the deposition of a thin-film material onto a prefabricated TEM wedge substrate. The latter technique is rapid and straightforward enough for inclusion in standard deposition techniques used for semiconductors or devices. To validate these methods, both techniques were applied to the same UNCD film to extract line contours which were used to calculate statistical roughness parameters. Both techniques were demonstrated to yield equally reliable statistics, and the thickness of the prepared sample was shown to have no 
consistent effect on measured results. For confirmation, simulated surfaces were created with selfaffine, randomly rough surfaces. The simulations yielded nearly identical outcomes and demonstrated an explanation for the lack of dependence on thickness: increasing the sample thickness changes the particular features that are sampled, but not the statistical character of those features.

\section{Acknowledgements}

Sample preparation was performed in the Fischione Instruments Electron Microscopy Sample Preparation Laboratory at the University of Pittsburgh. The authors acknowledge the use of the Nanoscale Fabrication and Characterization Facility (NFCF) in the Petersen Institute of Nano Science and Engineering (PINSE). LP acknowledges partial funding by the Deutsche Forschungsgemeinschaft (grant PA 2023/2). Funding for TDBJ and the University of Pittsburgh work was provided by the National Science Foundation under award number CMMI- 1727378. 


\section{References}

[1] Archard J F 1957 Elastic deformation and the laws of friction Proc. R. Soc. London Ser. A-Mathematical Phys. Eng. Sci. 243 190-205

[2] Sayles R S and Thomas T R 1978 Surface topography as a nonstationary random process Nature 271 431-4

[3] Greenwood J A and Williamson J B P 1966 Contact of Nominally Flat Surfaces Proc. R. Soc. A Math. Phys. Eng. Sci. 295 300-19

[4] Bush A W, Gibson R D and Thomas T R 1975 The elastic contact of a rough surface Wear 35 87-111

[5] Persson B N J, Albohr O, Tartaglino U, Volokitin A I and Tosatti E 2005 On the nature of surface roughness with application to contact mechanics, sealing, rubber friction and adhesion J. Phys. Condens. Matter 17 R1-62

[6] Campañá C, Müser M H and Robbins M O 2008 Elastic contact between self-affine surfaces: comparison of numerical stress and contact correlation functions with analytic predictions J. Phys. Condens. Matter 20354013

[7] Stover J C 1995 Optical scattering: measurement and analysis vol 2 (SPIE Press, Bellingham, Washington)

[8] Jacobs T D B, Junge T and Pastewka L 2017 Quantitative characterization of surface topography using spectral analysis Surf. Topogr. Metrol. Prop. 5013001

[9] Carim A H and Sinclair R 1987 The Evolution of Si / SiO2 Interface Roughness $J$. Electrochem. Soc. 134 741-6

[10] Lim J, Hippalgaonkar K, Andrews S C, Majumdar A and Yang P 2012 Quantifying surface roughness effects on phonon transport in silicon nanowires Nano Lett 12 2475-82

[11] Blanc J, Buiocchi C J, Abrahams M S and Ham W E 1977 The Si/SiO2 interface examined by cross-sectional transmission electron microscopy Appl. Phys. Lett. 30 120-2

[12] Krivanek O L, Tsui D C, Sheng T T and Kamgar A 1978 A high-resolution electron microscopy study of the $\mathrm{Si}-\mathrm{SiO} 2$ Interface The Physics of SiO2 and Its Interfaces: Proceedings of the International Topical Conference on the Physics of SiO2 and Its Interfaces ed S T Pantelides (Yorktown Heights, NY: Elsevier)

[13] Goodnick S M, Ferry D K, Wilmsen C W, Liliental Z, Fathy D and Krivanek O L 1985 Surface roughness at the Si(100)-SiO2 interface Phys. Rev. B 32 8171-86

[14] Zhao Y, Matsumoto H, Sato T, Koyama S, Takenaka M and Takagi S 2010 A Novel Characterization Scheme of $\$ \backslash$ hbox $\{\mathrm{Si} / \mathrm{SiO}\}$ _ $\{2\} \$$ Interface Roughness for Surface Roughness Scattering-Limited Mobilities of Electrons and Holes in Unstrained-and Strained-Si MOSFETs IEEE Trans. Electron Devices 57 2057-66

[15] Pastewka L, Salzer R, Graff A, Altmann F and Moseler M 2009 Surface amorphization, sputter rate, and intrinsic stresses of silicon during low energy $\mathrm{Ga}+$ focused-ion beam milling Nucl. Instruments Methods Phys. Res. Sect. B Beam Interact. with Mater. Atoms $2673072-5$

[16] Williams D B and Carter C B 2009 Transmission Electron Microscopy: A Textbook for Materials Science (Springer)

[17] Strecker A, Bäder U, Kelsch M, Salzberger U, Sycha M, Gao M, Richter G and van Benthem K 2003 Progress in the Preparation of Cross-Sectional TEM Specimens by IonBeam Thinning Zeitschrift für Met. 94 290-7

[18] Zeng H, Konicek A R, Moldovan N, Mangolini F, Jacobs T D B, Wylie I, Arumugam P 
U, Siddiqui S, Carpick R W and Carlisle J A 2015 Boron-doped ultrananocrystalline diamond synthesized with an H-rich/Ar-lean gas system Carbon N. Y. 84 103-17

[19] Malis T, Cheng S C, and Egerton R F 1988 EELS log-ratio technique for specimen-thickness measurement in the TEM J. Elec. Microsc. Tech. 8 193-200

[20] Egerton R F 2011 Electron energy-loss spectroscopy in the electron microscope (Springer Science \& Business Media)

[21] Gujrati A, Khanal S R, Pastewka L and Jacobs T D B 2018 Combining TEM, AFM, and profilometry for quantitative topography characterization across all scales $\mathrm{ACS} A \mathrm{Apl}$. Mater. Interfaces $1029169-29178$

[22] Ramisetti S B, Campañá C, Anciaux G, Molinari J-F, Müser M H and Robbins M O 2011 The autocorrelation function for island areas on self-affine surfaces. J. Phys. Condens. Matter 23215004

[23] Church E L and Takacs P Z 1991 Effects of the nonvanishing tip size in mechanical profile measurements Proc. SPIE 1332, Optical Testing and Metrology III: Recent Advances in Industrial Optical Inspection vol 1332, ed C P Grover (International Society for Optics and Photonics) pp 504-14

[24] Persson B N J 2014 On the Fractal Dimension of Rough Surfaces Tribol. Lett. 54 99-106 


\title{
Supplementary Materials for "Small-scale surface topography characterization using transmission electron microscopy"
}

\author{
Authors: \\ Subarna R. Khanal, ${ }^{1}$ Abhijeet Gujrati, ${ }^{1}$ Sai Bharadwaj Vishnubhotla, ${ }^{1}$ Pawel Nowakowski, ${ }^{2}$ \\ Cecile S. Bonifacio, ${ }^{2}$ Lars Pastewka, ${ }^{3}$ Tevis D. B. Jacobs ${ }^{1 *}$
}

\section{Affiliations: \\ 1. Mechanical Engineering and Materials Science, University of Pittsburgh, Pittsburgh, PA \\ 2. Fischione Instruments, Export, PA \\ 3. Microsystems Engineering, University of Freiburg, Freiburg, Germany \\ * Corresponding author: tjacobs@pitt.edu}

\section{S-1. Additional examples of traced profiles at a variety of magnifications}

Figure 5 of the main text includes statistics from 168 TEM measurements on different samples, taken at a variety of different locations and magnifications. Several examples of these TEM images and traced profiles are shown in figures 4 and 6 of the main text. Figure S1 provides additional representative examples of these measurements, and demonstrates how the scan sizes and pixel sizes will vary with magnification of the image.

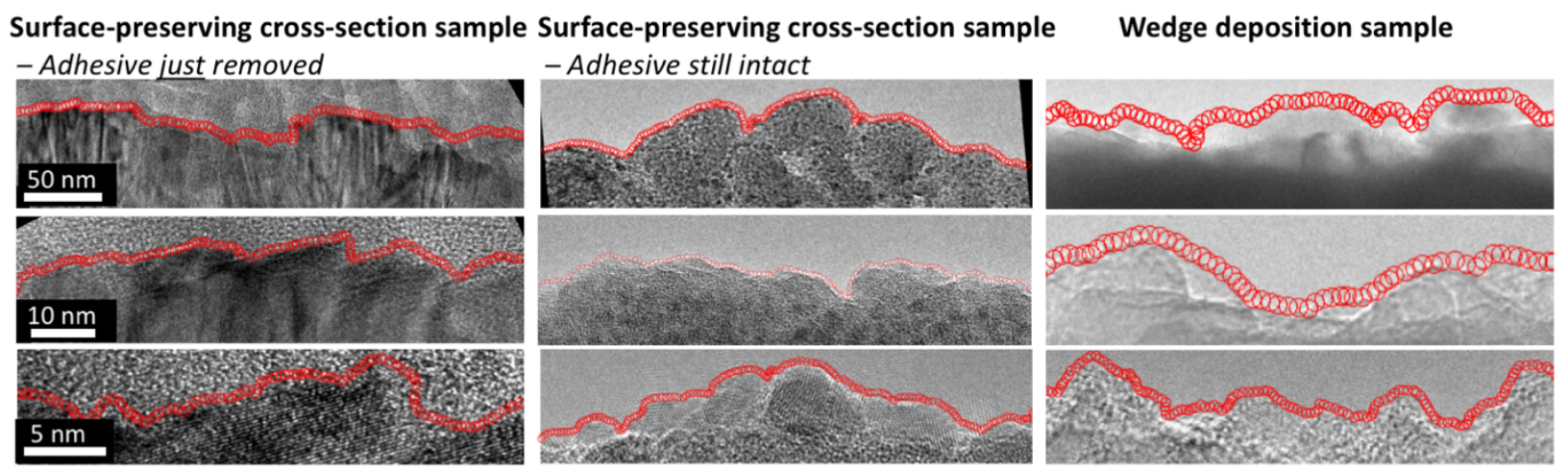

Figure S1. Additional TEM images and extracted profiles from the three different sample preparations (columns). Each row represents a different magnification for analysis; all images in the same row are presented with a common scale bar. 


\section{S-2. The effect of sample thickness on RMS slope and RMS curvature in TEM-measured topography}

In the main text (figure 6(d)), the RMS height is plotted to show that the sample thickness has no systematic effect. For space reasons, we omitted the plots of the other roughness parameters: RMS slope and RMS curvature. These metrics are shown in figure S2; they also show no systematic trends with sample thickness.
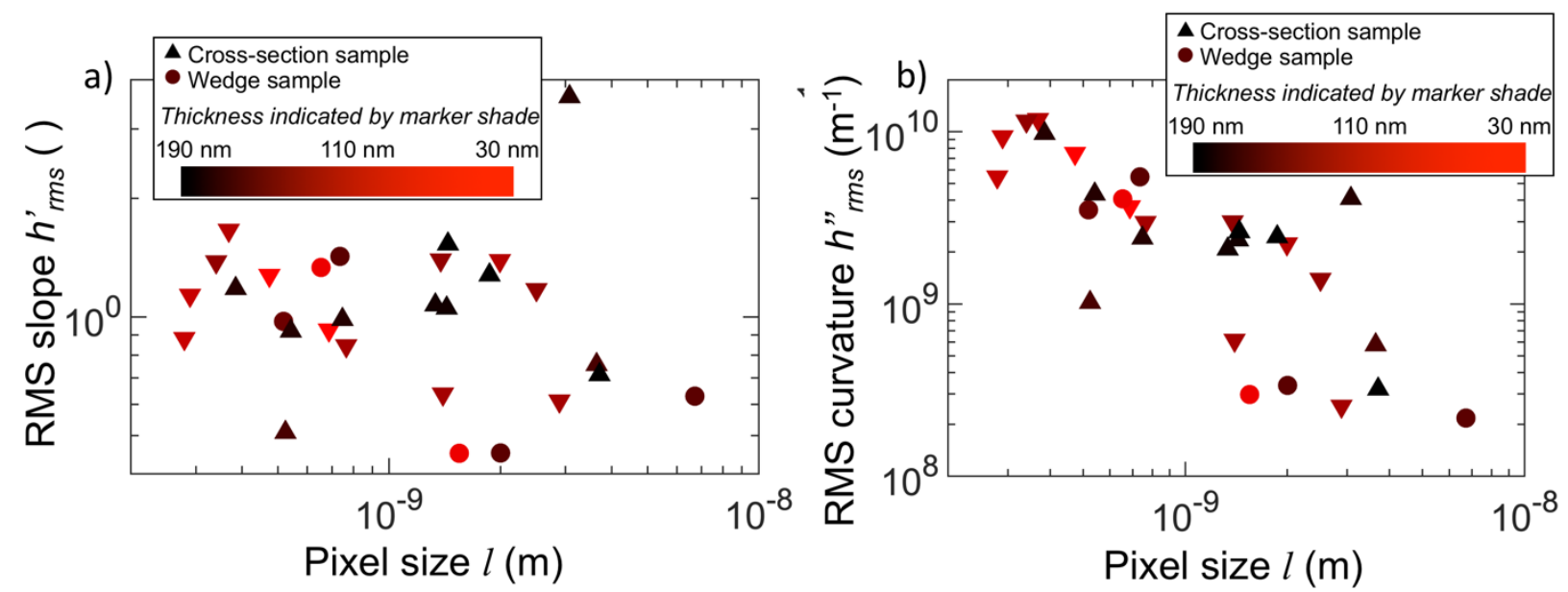

Figure S2. Small-scale roughness metrics demonstrate no systematic effect of thickness on results. The RMS slope (a) and RMS curvature (b) show trends with pixel size (as expected), but do not show any systematic trends with sample thickness. Here and in figure 6 of the main text, the sample thickness is indicated by the color of the marker. The preparation type is indicated by the marker shape, with right-side-up triangles indicating crosssection samples with the adhesive still intact and upside-down triangles indicating cross-section samples with the adhesive just removed.

\section{S-3. The effect of thickness on PSD for simulated samples with a large-wavelength roughness roll-off}

In the main text (figure 7(d) and (h)), the PSD is plotted for simulated samples with a smallwavelength roughness cutoff as a function of varying thickness (for simulated cross-section samples) and radius (for simulated wedge samples). In figure S3, the measured profiles and measured PSDs are plotted for simulated surfaces with a large-wavelength (low- $q$ ) roughness rolloff. The true PSD that was used to create these surfaces has a constant value at wavelengths larger than $20 \mathrm{~nm}\left(\lambda_{\text {roll-off }}=20 \mathrm{~nm}, q_{\text {roll-off }}=3.1 \times 10^{8} \mathrm{~m}^{-1}\right)$. In this case, there is no discernible effect of thickness in the small-scale (high- $q$ ) portion of the PSD, but there is a reduction in amplitude of the large-scale (low- $q$ ) portion. This reduction begins to manifest when the thickness is larger than $\lambda_{\text {roll-off. In the experimental measurements of the present paper, this reduction at low- } q \text { is not }}$ observed because the TEM is only being used to measure the fine-scale topography (less than $1 \mu \mathrm{m})$, which is smaller than the roll-off value for ultrananocrystalline diamond [1]. Furthermore, topography at scales larger than tens of $\mathrm{nm}$ can be measured faithfully using atomic force microscopy, as shown in [1], and is therefore not the focus of the present method. Nevertheless, 
we report the effect of this diminished amplitude of the PSD as yet another artifact that can be observed in the experimental measurement of PSDs, in combination with the ones reported in [2].
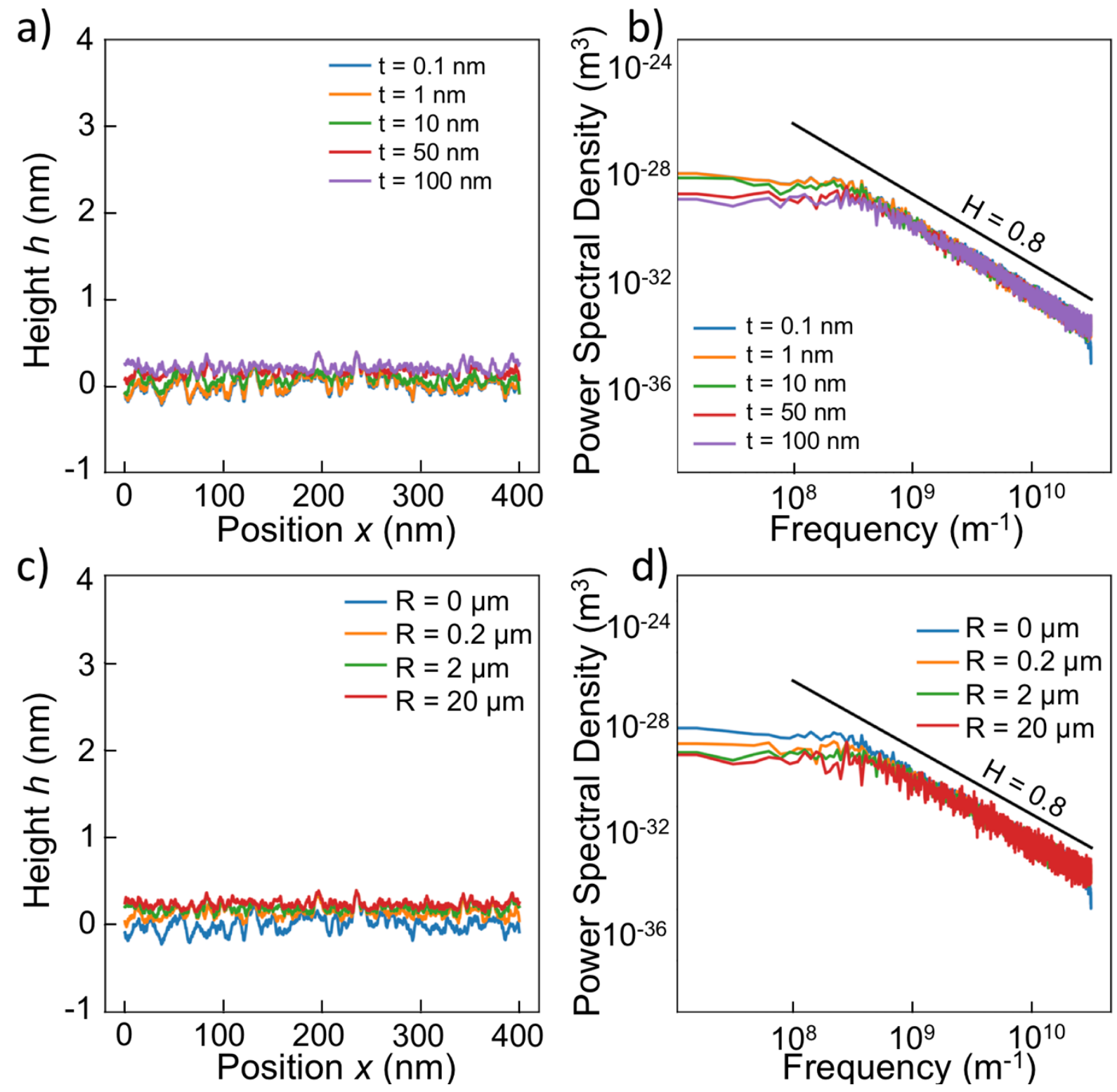

Figure S3. Emulation of the effect on the measured power-spectral density of a topography roll-off to white noise using computer-generated samples. Panels (a) and (b) show the results for cross-section samples of varying thickness $t$ while (c) and (d) show the results obtained for wedge samples of varying radius $R$. The initial powerspectral density has a roll-off at 20-nm wavelength. Panels (a) and (c) show the resulting line contours and panels (b) and (d) show the corresponding power-spectral densities. The small-scale (high-q) behavior is unaffected; the amplitude of the PSD in the large-scale (low-q) regime is reduced. 


\section{S-4. References}

[1] Gujrati A, Khanal S R, Pastewka L and Jacobs T D B 2018 Combining TEM, AFM, and profilometry for quantitative topography characterization across all scales ACS Appl. Mater. Interfaces 10 29169-29178

[2] Jacobs T D B, Junge T and Pastewka L 2017 Quantitative characterization of surface topography using spectral analysis Surf. Topogr. Metrol. Prop. 5013001 